\title{
Atomistic calculations of elastic properties of metallic fcc crystal surfaces
}

\author{
Vijay B. Shenoy* \\ Materials Research Centre, Indian Institute of Science, Bangalore 560 012, India
}

\begin{abstract}
Elastic properties of crystal surfaces are useful in understanding mechanical properties of nanostructures. This paper presents a fully nonlinear treatment of surface stress and surface elastic constants. A method for the determination of surface elastic properties from atomistic simulations is developed. This method is illustrated with examples of several crystal faces of some fcc metals modeled with embedded atom potentials. The key finding in this study is the importance of accounting for the additional relaxations of atoms at the crystal surface due to strain. Although these relaxations do not affect the values of surface stress (as had been determined in previous works), they have a profound effect on the surface elastic constants. Failure to account for these relaxations can lead to values of elastic constants that are incorrect not only in magnitude but also in sign. A possible method for the experimental determination of the surface elastic constants is outlined.
\end{abstract}

\section{INTRODUCTION}

The understanding of properties of surfaces of solid materials is important from both scientific and technological viewpoints. Surfaces of solids possess interesting properties owing to the fact that they possess atoms with fewer neighbors and consequently excess energy over atoms in the bulk. The properties of surfaces dominate processes ranging from intercellular transport in biological systems to the growth of thin films used in microelectronic devices.

An important property of solid surfaces is the surface stress. ${ }^{1,2}$ When a liquid surface is deformed, atoms from the bulk are free to migrate to the surface in such a manner that the number of atoms per unit area of the surface remains roughly a constant in the deformation process. Surface stress arises in solids due to the fact that as the solid deforms, atoms from the bulk are unable to move to occupy the surface. Surface stress has been shown to be responsible for many interesting effects on crystal surfaces. One such effect is the reconstruction ${ }^{3-7}$ of the close packed (111) surfaces of $\mathrm{Au}$ and Pt. This reconstruction has been attributed to a competition between surface stress and the interlayer interaction. ${ }^{2,8,9}$ The importance of surface stress has lead to several efforts in its theoretical determination. For example, surface stresses were evaluated using ab initio methods in semiconductors by Maede and Vanderbilt ${ }^{10}$ and in metals by Needs. ${ }^{11}$ More recently, Feibelman has investigated the nature of anisotropy of surface stress ${ }^{12}$ on certain fcc crystal surfaces and the effect of gas adsorption ${ }^{13}$ on surface stress. Empirical potentials have also been employed to study surface stresses in bcc and fcc crystals. ${ }^{14,15}$ Although the empirical methods generally underestimate the magnitude of the surface stress, they remain useful in understanding trends and developing concepts.

With the emergence of nanotechnology in the last decade, it is now possible to make structures and devices at nanometric scales. These "nanostructures" are characterized by a large surface-to-volume ratio. In this context, it is all the more important to understand properties of surfaces that make up the nanostructure. Indeed, nanostructures show "size-dependent" behavior. In a set of experiments, Gao et $a l .{ }^{16}$ studied the properties of multiwalled carbon nanotubes by exciting them with an external field and observing the amplitude response as a function of the excitation frequency. From the resonant frequency they calculated the elastic modulus of the tube using standard continuum mechanics and found that the calculated elastic modulus is a strong function of the diameter of the tube. In another set of experiments, Yang et al. ${ }^{17}$ found that the loss factor of ultrathin silicon cantilevers depends on the thickness of the cantilevers, an effect that they attributed to surface stress.

A simple model to explain the size dependence of the elastic rigidities of nanostructures was developed by Miller and Shenoy. ${ }^{18}$ The main premise of the model is that although the discrete atomistic nature of the material becomes important at smaller scales, the nanostructure can be modeled using continuum mechanics following the ansatz that "nanostructure = bulk + surface." It was shown that the differences between the actual rigidity $(D)$ of a nanostructure and that predicted by continuum mechanics $\left(D_{c}\right)$ can be expressed as

$$
\frac{D-D_{c}}{D_{c}}=A \frac{h_{0}}{a},
$$

where $A$ is a nondimensional constant that depends on the geometry of the structure, $a$ is the size scale of the structural element (for example, the cross-sectional width of a bar), and $h_{0}$ is a material length that is the ratio of the surface elastic constant of the bounding surfaces of the structure and the bulk elastic constant. Thus the size dependence of the rigidities can be predicted by obtaining the material parameter $h_{0}$ and the nondimensional constant $A$. Typically, $h_{0}$ can be obtained from atomistic simulations that determine the value of the surface and bulk elastic constants. The constant $A$, which depends only on the geometry (cross section) of the structure, can be calculated analytically. Thus the need for full scale atomistic simulations of nanostructures is obviated. This model was used to study the elastic properties of nanosized bars, plates, and beams, ${ }^{18}$ and also to study the torsion of bars. ${ }^{19}$ In both cases, excellent agreement was obtained 
between theory and atomistic simulations. The main point brought out by the above cited work is that the elastic response of nanostructures depends on the surface elastic con$\operatorname{stant}(s)$.

The idea of the surface elastic constant is now illustrated in a simple one-dimensional setting. Let $\tau$ denote the surface stress $^{20}$ which is related to the surface energy $\gamma$ via

$$
\tau(\epsilon)=\gamma+\frac{\partial \gamma}{\partial \epsilon}
$$

where $\epsilon$ is the strain. Clearly, the surface stress depends on the strain since the surface energy depends on the strain. What is usually referred to as surface stress and values reported ${ }^{10-15}$ is the value of the surface stress at zero strain, i.e., $\tau(0)=\tau^{0}$. The surface elastic constant $S$ is a measure of how the surface stress changes with strain, i.e.,

$$
\tau(\epsilon)=\tau^{0}+S \epsilon
$$

or, alternatively,

$$
S=\left.\frac{\partial \tau}{\partial \epsilon}\right|_{\epsilon=0} .
$$

The effect of surfaces on the biaxial modulus of thin films and multilayers was first noted by Streitz et al. ${ }^{21,22}$ These authors developed a scaling relation similar to Eq. (1), but did not introduce surface elastic constants explicitly. In addition, their analysis is restricted to biaxial deformations with linear kinematics. Although surface elastic constants were introduced and calculated in previous works ${ }^{18,19}$ with a view a validating Eq. (1), a systematic study of these quantities is yet to be performed. The aim of this paper is twofold. First, to develop a general nonlinear framework for surface mechanics in which the surface elastic constants are introduced, and second, to establish a procedure for calculating these quantities from atomistic simulations. While ab initio methods provide the most accurate results (in terms of numerical values), the present calculations are performed using the embedded atom method. ${ }^{23,24}$ The advantage of this method is that it allows for an efficient study of a large number of crystal surfaces and materials and is therefore ideally suited to achieve the second objective of this paper as stated above. Attention is restricted to fcc crystals.

The paper is organized as follows. Section II contains the theoretical background to the mechanical properties of surfaces. The method for obtaining the surface elastic constants within the framework of atomistic simulations is outlined in Sec. III. The results are presented and discussed in Sec. IV. The last section also contains a discussion of possible future directions of investigation.

\section{MECHANICAL PROPERTIES OF CRYSTAL SURFACES: THEORY}

The mechanics of nanosized bodies requires consideration of both the bulk and surface effects. The effects of terminating a solid at a surface may extend several atomic layers below the terminating layer. The aim of the theoretical effort in understanding size-dependent properties of

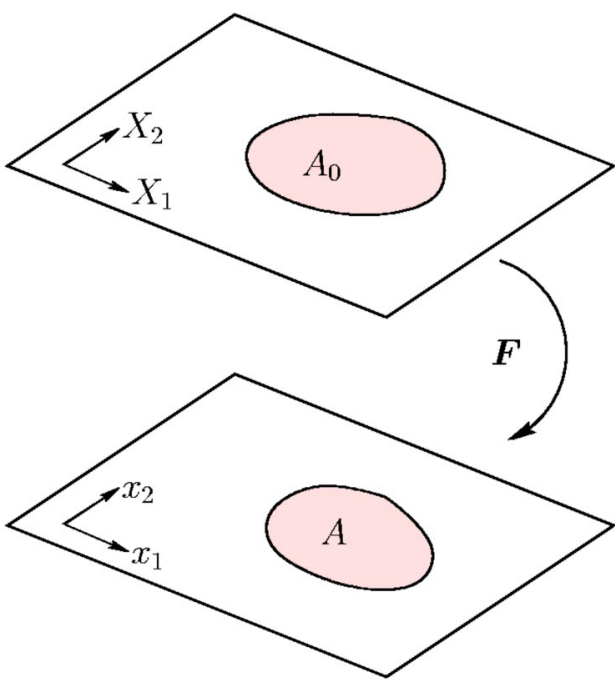

FIG. 1. Schematic of a flat surface undergoing homogeneous deformation. The deformation leaves the surface flat.

nanostructures ${ }^{18,19}$ is to develop simple continuum field theories that include the effect of surfaces. To this end, the nanosized body is considered to be made up of "bulk" and a bounding "surface" in a continuum theory. This bounding surface in the continuum theory is used to mathematically represent the actual atomic surface of the solid including the layers below the terminating layer where the effects of the termination are felt. This is achieved in two steps. First is to develop a fully nonlinear continuum theory of surface deformation and energetics. The key outcome of this step is the description of the surface mechanics via surface stress and elastic modulus tensors; this is described in this section. The second step is the calculation of the constitutive parameters, namely the surface stresses and elastic constants, which will include effects of layers below the terminating layer, from an atomistic simulation. This second step is taken up in the next section.

Nonlinear mechanics of a bulk solid is well known. ${ }^{25} \mathrm{Me}-$ chanics of surface deformation is considered in some detail by Haiss ${ }^{1}$ based on linear kinematics. The analysis here is based on nonlinear kinematics and a complete development is presented for the sake of clarity. The flat surface considered is described by coordinates $\left(X_{1}, X_{2}\right)$ in its undeformed state (Fig. 1). The surface undergoes homogeneous deformation described by the deformation gradient tensor ${ }^{25} \boldsymbol{F}$ whose components are denoted by $F_{i j}$. The point $\left(X_{1}, X_{2}\right)$ in the undeformed system is mapped to $\left(x_{1}, x_{2}\right)$ by $\boldsymbol{F}$ (Fig. 1), i.e.,

$$
x_{i}=F_{i j} X_{j}
$$

The components of the Green-Lagrange strain tensor $\epsilon$ are related to the deformation gradient tensor via

$$
\epsilon_{i j}=\frac{1}{2}\left(F_{k i} F_{k j}-\delta_{i j}\right)
$$

where $\delta_{i j}$ is the Kronecker delta symbol. 
Under the action of the homogeneous deformation $\boldsymbol{F}$, a patch with area $A_{0}$ is mapped to a patch with area $A$. The deformed area $A$ is related to the area $A_{0}$ via

$$
A=A_{0} \operatorname{det} \boldsymbol{F}=A_{0} \sqrt{\operatorname{det}(\boldsymbol{I}+2 \boldsymbol{\epsilon})},
$$

where $\boldsymbol{I}$ is the identity tensor. Two results to be used in the developments below are recorded. First is the derivative of the area $A$ with respect to the strain tensor components,

$$
\frac{\partial A}{\partial \epsilon_{i j}}=A\left[(I+2 \epsilon)^{-1}\right]_{i j}
$$

where $\left[(I+2 \epsilon)^{-1}\right]_{i j}$ denotes the $i j$ component of the tensor $(I+2 \epsilon)^{-1}$. Second is the derivative of the components of the tensor $(I+2 \epsilon)^{-1}$ with respect to the components of the strain tensor

$$
\frac{\partial\left[(I+2 \epsilon)^{-1}\right]_{i j}}{\partial \epsilon_{k l}}=2\left[(I+2 \epsilon)^{-1}\right]_{i k}\left[(I+2 \epsilon)^{-1}\right]_{j l} .
$$

The surface is assumed to posses an energy density $\gamma$ which is the energy per unit deformed area. Evidently $\gamma$ depends on the strain tensor $\epsilon$. With this definition of strain, the total energy $E^{s}$ in the patch $A$ can be written as

$$
E^{s}=A \gamma(\epsilon)
$$

To obtain an expression for surface stress, the strain state in incremented to $\epsilon+\delta \epsilon$ from $\epsilon$ and the change in total energy is evaluated using Eq. (8):

$$
\delta E^{s}=\left(\frac{\partial A}{\partial \epsilon_{i j}} \gamma+A \frac{\partial \gamma}{\partial \epsilon_{i j}}\right) \delta \epsilon_{i j}=A\left(\gamma\left[(I+2 \epsilon)^{-1}\right]_{i j}+\frac{\partial \gamma}{\partial \epsilon_{i j}}\right) \delta \epsilon_{i j} .
$$

The surface stress tensor $\tau$ (with components $\tau_{i j}$ ) is defined as the work conjugate of $\delta \epsilon_{i j}$, i.e.,

$$
\delta E^{s}=A \tau_{i j} \delta \epsilon_{i j} \Rightarrow \tau_{i j}=\frac{1}{A} \frac{\partial E^{s}}{\partial \epsilon_{i j}}=\left(\gamma\left[(I+2 \epsilon)^{-1}\right]_{i j}+\frac{\partial \gamma}{\partial \epsilon_{i j}}\right)
$$

The expression (12) is the definition consistent with nonlinear kinematics and the surface stress clearly depends on the strain. When Eq. (12) is evaluated at the unstrained configuration, i.e., when $\epsilon_{i j}=0$, the result above agrees with the previous definition of surface stress ${ }^{1,2,20}$

$$
\tau_{i j}^{0}=\left(\frac{1}{A} \frac{\partial E^{s}}{\partial \epsilon_{i j}}\right)_{\boldsymbol{\epsilon}=\mathbf{0}}=\left(\gamma \delta_{i j}+\frac{\partial \gamma}{\partial \boldsymbol{\epsilon}_{i j}}\right)_{\boldsymbol{\epsilon = 0}} .
$$

The surface elastic constants determine the change in the surface stress with strain. Thus the surface stress $\tau_{i j}$ is written as

$$
\tau_{i j} \approx \tau_{i j}^{0}+S_{i j k l} \epsilon_{k l},
$$

where $S_{i j k l}$ is the fourth order surface elastic tensor. An alternative definition of the surface elastic that is consistent with Eq. (14) is

$$
S_{i j k l}=\left.\frac{\partial \tau_{i j}}{\partial \epsilon_{k l}}\right|_{\boldsymbol{\epsilon}=\mathbf{0}}
$$

From Eqs. (12) and (15), it is easily shown using Eq. (8) that

\begin{tabular}{|c|c|c|c|}
\hline $\begin{array}{l}\text { Plane } \\
\text { point group }(\mathrm{s})\end{array}$ & $\begin{array}{l}\text { Number of independent } \\
\text { elastic constants }\end{array}$ & $\begin{array}{l}\text { Independent } \\
\text { elastic constants }\end{array}$ & $\begin{array}{l}\text { Symmetry imposed } \\
\text { constraint relations }\end{array}$ \\
\hline 1,2 & 9 & All & Nil \\
\hline $\mathrm{m}, 2 \mathrm{~mm}$ & 5 & $\begin{array}{c}S_{1111}, S_{1122}, S_{1212} \\
S_{2211}, S_{2222}\end{array}$ & $S_{1112}=S_{1211}=S_{1222}=S_{2212}=0$ \\
\hline 4 & 5 & $\begin{array}{c}S_{1111}, S_{1112}, S_{1211} \\
S_{1212}, S_{1122}\end{array}$ & $\begin{array}{l}S_{1222}=-S_{1211}, S_{2211}=S_{1122} \\
S_{2212}=-S_{1112}, S_{2222}=S_{1111}\end{array}$ \\
\hline $4 \mathrm{~mm}$ & 3 & $S_{1111}, S_{1122}, S_{1212}$ & $\begin{array}{c}S_{1112}=S_{1211}=S_{1222}=S_{2212}=0 \\
S_{2211}=S_{1122}, S_{2222}=S_{1111}\end{array}$ \\
\hline 3,6 & 3 & $S_{1111}, S_{1112}, S_{1122}$ & $\begin{array}{c}S_{1211}=-S_{1112}, 2 S_{1212}=S_{1111}-S_{1122} \\
S_{1222}=S_{1112}, S_{2211}=S_{1122}, \\
S_{2212}=-S_{1112}, S_{2222}=S_{1111}\end{array}$ \\
\hline $3 \mathrm{~m}, 6 \mathrm{~mm}$ & 2 & $S_{1111}, S_{1122}$ & $\begin{array}{c}S_{1112}=S_{1211}=S_{1222}=S_{2212}=0 \\
2 S_{1212}=S_{1111}-S_{1122} \\
S_{2211}=S_{1122}, S_{2222}=S_{1111}\end{array}$ \\
\hline
\end{tabular}

$$
S_{i j k l}=\left.\frac{1}{A} \frac{\partial^{2} E^{s}}{\partial \epsilon_{i j} \partial \epsilon_{k l}}\right|_{\boldsymbol{\epsilon}=\mathbf{0}}-\tau_{i j}^{0} \delta_{k l}
$$

and in terms of surface energy [on using Eq. (9)] as

$$
S_{i j k l}=\left.\left(2 \gamma \delta_{i k} \delta_{j l}+\delta_{i j} \frac{\partial \gamma}{\partial \epsilon_{k l}}+\frac{\partial^{2} \gamma}{\partial \epsilon_{i j} \partial \epsilon_{k l}}\right)\right|_{\boldsymbol{\epsilon = 0}} .
$$

The surface elastic constant tensor possesses the following symmetries with respect to exchange of indices:

TABLE I. Independent surface elastic constants for different plane point groups. 


$$
\begin{aligned}
& S_{j i k l}=S_{i j k l}, \\
& S_{i j l k}=S_{i j k l} .
\end{aligned}
$$

Notably, $S_{i j k l} \neq S_{k l i j}$, a symmetry that is present in the bulk elastic tensor. There are, therefore, a total of nine independent possible elastic constants for a crystal surface which are taken to be $S_{1111}, S_{1112}, S_{1122}, S_{1211}, S_{1212}, S_{1222}, S_{2211}, S_{2212}$, and $S_{2222}$. Further reduction in the number of independent elastic constants can is brought about by the presence of geometric symmetry in the crystal surface as shown in Table I.

In contrast to the bulk elastic tensor, the surface elastic tensor $S_{i j k l}$ need not be positive definite, i.e., the quadratic form $S_{i j k l} \epsilon_{i j} \epsilon_{k l}$ need not be non-negative. At first glance this may suggest a violation of basic thermodynamic postulates. It must be noted that the positive definiteness of the bulk elastic modulus tensor which guarantees the stability of the solid cannot be applied to the surface elastic tensor. This is due to the fact that a surface cannot exist independent of the bulk, and the total energy (bulk + surface) that needs to satisfy the positive definiteness condition. This property of the surface elastic modulus can lead to interesting consequences such as fall in the rigidity of a nanostructure ${ }^{18}$ with size.

\section{CALCULATION OF SURFACE ELASTIC CONSTANTS}

One of the objectives of this work is to establish a method to obtain surface elastic constants from atomistic calculations. While $a b$ initio electronic structure methods ${ }^{26}$ are the most reliable in terms of numerical values, approximate total energy descriptions such as the embedded atom method ${ }^{23,24}$ provide for efficient computations. Since the purpose of this work is not the accurate determination of numerical values of surface elastic constants, but to establish consistent methods for the determination of the surface elastic constants from a total energy description, the embedded atom method is adopted. It must be emphasized that the methods presented here can be applied to any atomistic formulation.

The total energy in the embedded atom formalism ${ }^{23,24}$ depends on the positions of the atoms (restricted here to solids with single species of atoms) via

$$
\begin{gathered}
E^{t o t}=\frac{1}{2} \sum_{\alpha} \sum_{\beta \neq \alpha} \phi\left(r_{\alpha \beta}\right)+\sum_{\alpha} F\left(\rho_{\alpha}\right), \\
\rho_{\alpha}=\sum_{\beta \neq \alpha} \rho\left(r_{\alpha \beta}\right),
\end{gathered}
$$

where $\alpha$ and $\beta$ are indices that run over the atoms, $r_{\alpha \beta}$ is the distance between atom $\alpha$ and $\beta, \phi(r)$ is a function that describes a pairwise interaction between the atoms, $\rho(r)$ is the electron density function, $\rho_{\alpha}$ is the electron density at the site of atom $\alpha$, and $F$ is the embedding function. The embedded atom method accounts for the many body effects in an approximate manner via the nonlinear embedding function. It must be noted that the total energy $E^{\text {tot }}$ in Eq. (20) is taken to

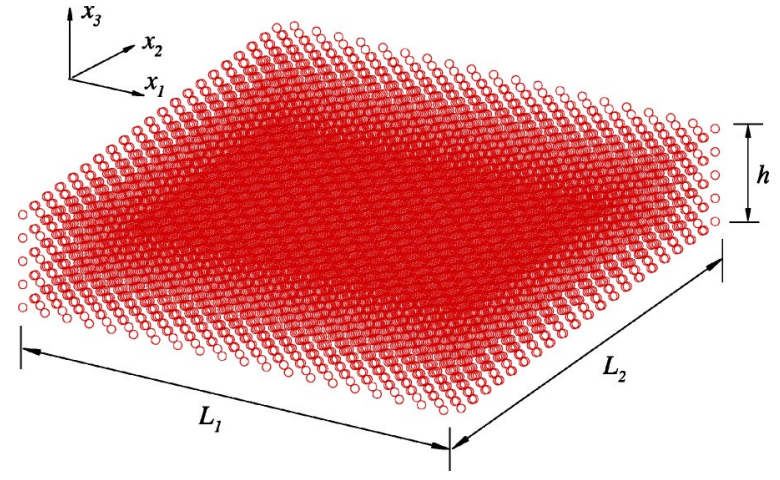

FIG. 2. A simulation cell used to determine surface elastic properties consists of a slab of thickness $h$. Periodic boundary conditions are used in the 1 and 2 directions.

be the additional energy with respect to the undistorted lattice, i.e., the energy reference is chosen such that an undistorted lattice will have zero energy per atom.

To calculate the surface elastic properties, a simulation cell consisting of atoms as shown in Fig. 2 is constructed. Periodic boundary conditions are used in the " 1 " and "2" directions while the box is bounded in the third direction by free surfaces whose properties are to be studied. An important parameter is the thickness $h$ of the slab. This thickness is determined by a Gibbs dividing surface construction ${ }^{27}$ based on total mass. Thus $h$ is chosen such that the volume of the cell $\left(L_{1} L_{2} h\right)$ in its undistorted configuration multiplied by the density of the bulk solid is equal to the total mass of the atoms in the box. The lengths $L_{1}$ and $L_{2}$ are typically chosen to be five to six times the repeat distance in the appropriate crystallographic direction. It is found that the calculated surface elastic properties do not depend on the particular choice of $L_{1}$ and $L_{2}$.

To determine the unstrained (reference) configuration of the surface, the following method is adopted. Atoms are stacked in positions as if in a perfect crystal in the simulation box shown in Fig. 2. An energy minimization is performed such that the atoms at the surface relax to their equilibrium positions. This relaxed configuration of the surface is taken as the reference configuration defined in the previous section (Fig. 1).

There are two possible methods for evaluating the surface stress $\tau_{i j}^{0}$ and elastic tensor $S_{i j k l}$. The first method, adopted in previous calculations ${ }^{14,28}$ (in the case of surface stress), is to evaluate the expression for surface stress in terms of the strain derivative of the total surface energy given in Eq. (12). The strain derivative of the surface energy is derived analytically. In the case of the embedded atom method, the strain derivative of the total energy is given by

$$
\frac{\partial E^{t o t}}{\partial \epsilon_{i j}}=\sum_{\alpha} \sum_{\beta \neq \alpha}\left(\frac{1}{2} \phi^{\prime}\left(r_{\alpha \beta}\right)+F^{\prime}\left(\rho_{\alpha}\right) \rho^{\prime}\left(r_{\alpha \beta}\right)\right) \frac{r_{\alpha \beta}^{i} r_{\alpha \beta}^{j}}{r_{\alpha \beta}},
$$

where the primes denote derivatives. In case of $a b$ initio electronic structure methods, a conceptually similar expression is used. ${ }^{28,29}$ For the developments that follow it is im- 
portant to understand how Eq. (21) is derived. To obtain the strain derivative of the total energy, the positions of all atoms are modified by the applied homogeneous deformation gradient. Stated in another way, if $\boldsymbol{r}$ is the position vector of an atom in the reference configuration, its position is changed to $r_{i} \rightarrow F_{i j} r_{j}$ under the action of the homogeneous deformation gradient. The total energy $\left[E^{t o t}(\boldsymbol{\epsilon})\right]$ is evaluated in this configuration where the atoms are placed in the positions dictated by the homogeneous deformation gradient. The expression (21) is obtained as $\lim _{\epsilon_{i j} \rightarrow 0}\left\{\left[E^{\text {tot }}(\boldsymbol{\epsilon})-E^{\text {tot }}(r e f)\right] / \epsilon_{i j}\right\}$. The derivation of the stress expression in the case of $a b$ initio methods ${ }^{28,29}$ is also similar in that the ground state electronic wave functions are "stretched" by the strain tensor and the difference in the energy is obtained and the limiting process described above provides the strain derivative. The surface elastic modulus can also be obtained using Eq. (16), in the case of the embedded atom method, from the second derivative of the total energy:

$$
\begin{aligned}
\frac{\partial^{2} E^{t o t}}{\partial \epsilon_{i j} \partial \epsilon_{k l}}= & \sum_{\alpha} \sum_{\beta \neq \alpha}\left[\frac{1}{2}\left(\phi^{\prime \prime}\left(r_{\alpha \beta}\right)-\frac{\phi^{\prime}\left(r_{\alpha \beta}\right)}{r_{\alpha \beta}}\right)-F^{\prime}\left(\rho_{\alpha}\right)\right. \\
& \left.\times\left(\rho^{\prime \prime}\left(r_{\alpha \beta}\right)-\frac{\rho^{\prime}\left(r_{\alpha \beta}\right)}{r_{\alpha \beta}}\right)\right] \frac{r_{\alpha \beta}^{i} r_{\alpha \beta}^{j} r_{\alpha \beta}^{k} r_{\alpha \beta}^{l}}{r_{\alpha \beta}^{2}}+\sum_{\alpha} F^{\prime \prime}\left(\rho_{\alpha}\right) \\
& \times\left(\sum_{\beta} \rho^{\prime}\left(r_{\alpha \beta}\right) \frac{r_{\alpha \beta}^{i} r_{\alpha \beta}^{j}}{r_{\alpha \beta}}\right)\left(\sum_{\beta} \rho^{\prime}\left(r_{\alpha \beta}\right) \frac{r_{\alpha \beta}^{k} r_{\alpha \beta}^{l}}{r_{\alpha \beta}}\right) .
\end{aligned}
$$

It must be noted that the second derivative of the total energy contains both bulk and surface contributions. The surface contribution can be isolated using the method described below.

The second method to obtain the surface stress tensor and elastic tensor is to obtain the total surface energy as a function of strain and calculate the required quantities by numerical differentiation. When the slab in Fig. 2 is strained, the totalenergy of the system is made of two components, the strain energy in the bulk and the surface energy. The surface energy has to be isolated from the total energy. If $L_{1} L_{2}\left(L_{1}\right.$ and $L_{2}$ are taken in the strained state) is taken as $A$, the area of the surface, the total energy can be written as

$$
E^{t o t}=2 \underbrace{2 A \gamma(\boldsymbol{\epsilon})}_{E^{s}}+\underbrace{h A W(\boldsymbol{\epsilon})}_{E^{b}},
$$

where $W(\boldsymbol{\epsilon})$ is the bulk strain energy density and $E^{b}$ is the total energy in the bulk. Thus, for a given state of strain, the total energy depends linearly on the thickness $h$ (determined by the Gibbs construction) of the slab.

Indeed, calculations (Fig. 3) show that the linear relationship is very closely followed. For a given strain state, surface energy $E^{s}$ can therefore be determined by obtaining the total energy as a function of thickness. A linear fit (3) is made to the $E^{\text {tot }}$ vs $h$ data, the intercept of which is twice the surface energy. This procedure has proved to be very robust. An important check to test the accuracy of the fit is to determine the surface energy based on different sets of values of $h$. The present calculations show that in every case reported here, the values were to within one part in $10^{6}$ of each other. The present method, therefore, provides a very accurate method

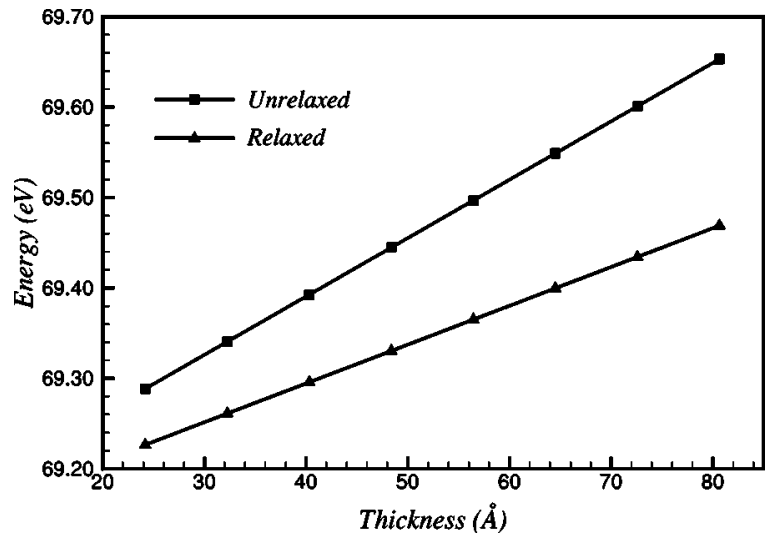

FIG. 3. Dependence of the total energy of the system on the thickness of the slab. Results shown are for the case of $\mathrm{Al}(001)$ surface at a strain of $\epsilon_{11}=0.003, \epsilon_{12}=0.003, \epsilon_{22}=0.003$ using a slab (see Fig. 2) with $L_{1}=L_{2}=24.192 \AA$. For the values of thicknesses and the strain state, the majority contribution to the total energy $E^{\text {tot }}$ arises from $E^{s}$ [of Eq. (23)] as the bulk elastic energy works out only to $2.33 \times 10^{-4} \mathrm{eV} /$ atom.

for determining the total surface energy $E^{s}$ as a function of strain. Once the total surface energy $E^{s}$ is obtained, expressions (13) and (16) are used to, respectively, obtain the surface stress and elastic tensors by numerical differentiation.

The two methods outlined above have one very important difference which proves to be crucial in the correct calculation of the surface elastic tensor. In the second method, the system is fully relaxed on application of the strain. The relaxation entails a Poisson contraction of the entire set of atoms in the 3 direction, and rearrangements of surface atoms to their new equilibrium positions. In the first method where analytical derivatives are used to obtain the required quantities, the effects due to the additional surface relaxations due to strain are not accounted for. Figure 3 shows the total energy as a function of the thickness for both the relaxed and unrelaxed cases, and the importance of relaxation is evident. A more vivid illustration of this effect is shown in Fig. 4 where the dependence of both relaxed and

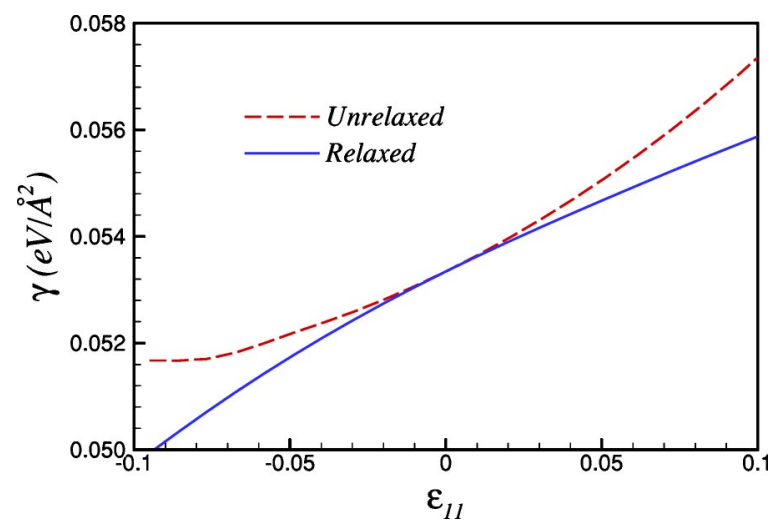

FIG. 4. Surface energy of the (001) surface of Al (Ref. 30) as a function of uniaxial strain in the [100] direction. The curvature of the relaxed and unrelaxed surface energies are different. Relaxation here refers to the additional rearrangements of surface atoms due to application of strain. 
TABLE II. Surface stress tensor of the (001) crystal face. The "1" direction corresponds to [100] and "2" corresponds to [010]. "UR" stands for unrelaxed results and " $R$ " denotes values for relaxed surfaces. Relaxation here denotes additional relaxation due to strain.

\begin{tabular}{|c|c|c|c|c|c|c|c|}
\hline \multirow[b]{2}{*}{ Element } & \multirow[b]{2}{*}{$\gamma\left(\mathrm{eV} / \AA^{2}\right)$} & \multicolumn{2}{|c|}{$\tau_{11}^{0}\left(\mathrm{eV} / \AA^{2}\right)$} & \multicolumn{2}{|c|}{$\tau_{22}^{0}(\mathrm{eV} / \AA)$} & \multicolumn{2}{|c|}{$\tau_{12}^{0}\left(\mathrm{eV} / \AA^{2}\right)$} \\
\hline & & UR & $\mathrm{R}$ & UR & $\mathrm{R}$ & UR & $\mathrm{R}$ \\
\hline $\mathrm{Ag}$ & 0.0543 & 0.0556 & 0.0556 & 0.0556 & 0.0556 & 0.0000 & 0.0000 \\
\hline $\mathrm{Al}(\mathrm{EA})$ & 0.0588 & 0.0355 & 0.0355 & 0.0355 & 0.0355 & 0.0000 & 0.0000 \\
\hline $\mathrm{Al}(\mathrm{V})$ & 0.0533 & 0.0822 & 0.0822 & 0.0822 & 0.0822 & 0.0000 & 0.0000 \\
\hline $\mathrm{Au}$ & 0.0498 & 0.0877 & 0.0877 & 0.0877 & 0.0877 & 0.0000 & 0.0000 \\
\hline $\mathrm{Cu}$ & 0.0828 & 0.0649 & 0.0649 & 0.0649 & 0.0649 & 0.0000 & 0.0000 \\
\hline $\mathrm{Ni}$ & 0.0956 & 0.0442 & 0.0442 & 0.0442 & 0.0442 & 0.0000 & 0.0000 \\
\hline $\mathrm{Pd}$ & 0.0807 & 0.1052 & 0.1052 & 0.1052 & 0.1052 & 0.0000 & 0.0000 \\
\hline $\mathrm{Pt}$ & 0.0880 & 0.1426 & 0.1426 & 0.1426 & 0.1426 & 0.0000 & 0.0000 \\
\hline
\end{tabular}

unrelaxed surface energies on strain is plotted. It is evident that although the slopes are equal in both cases when strain is zero, the curvatures of the two are vastly different. Thus the first method for the calculation of the surface stress tensor and the surface elastic modulus tensor based on the analytical derivative pertains to the unrelaxed case where additional surface relaxations due to the strain are neglected, while the second method based on the numerical derivative of the total surface energy accounts for surface relaxations. It is found in the calculations here that the additional surface relaxations do not affect the value of the surface stress $\tau_{i j}^{0}$ in a significant manner, but can have profound effect on the surface elastic tensor in that even the sign can be different when calculated in these two different ways. Needless to state that the second method where additional relaxations are accounted for corresponds to the correct physical situation.

\section{RESULTS AND CONCLUSIONS}

This section contains results of the calculations performed to determine the surface stress tensor and the surface elastic constants based on embedded atom simulations. Attention is restricted to fcc crystals. Four different surface orientations viz. (001), (110), (111), and (112) are studied. The elements considered are $\mathrm{Ag}, \mathrm{Al}, \mathrm{Au}, \mathrm{Cu}, \mathrm{Ni}, \mathrm{Pd}$, and Pt. To study the effect of the particular embedded atom potentials $\mathrm{Al}$ is treated with two different potentials. When treated with embedded atom potentials of Ercolessi and Adams ${ }^{30}$ derived by a force matching method from $a b$ initio calculations, Al is denoted as $\mathrm{Al}(\mathrm{EA})$. The second embedded atom potential chosen is that of $\mathrm{Al}$ obtained by $\operatorname{Voter}^{31}$ denoted as $\mathrm{Al}(\mathrm{V})$. All the remaining elements are treated using the embedded atom potentials developed by $\mathrm{Oh}$ and Johnson. ${ }^{32}$ These potentials have the same functional forms and the parameters in these functions are determined by fit to measured properties.

The total surface energy $E^{s}$ is determined using the above procedure for several values of strains; strains $\left(\epsilon_{11}, \epsilon_{22}, \epsilon_{12}\right)$ are each varied from -0.003 to 0.003 in steps of 0.001 and $E^{s}$ is determined for each set of values. A quartic polynomial in $\epsilon_{11}, \epsilon_{22}$, and $\epsilon_{12}$ is fit to this data and the required derivatives are extracted. The process of determination of $E^{s}$ is carried out both without allowing for additional relaxations and allowing for relaxations. In the former case where relaxations are not allowed, the results are compared with those using the analytic formulas and agreement to several significant figures is found confirming the correctness of the procedure.

\section{A. (001) crystal surface}

The (001) crystal face in fcc crystals belongs to the plane point group $\mathbf{4 m m}$. Thus the surface stresses are isotropic,

TABLE III. Surface elastic modulus tensor of the (001) crystal face. The "1" direction corresponds to [100] and "2" corresponds to [010]. "UR" stands for unrelaxed results and "R" denotes values for relaxed surfaces. Relaxation here denotes additional relaxation due to strain.

\begin{tabular}{|c|c|c|c|c|c|c|c|c|c|c|}
\hline \multirow[b]{2}{*}{ Element } & \multicolumn{2}{|c|}{$S_{1111}\left(\mathrm{eV} / \AA^{2}\right)$} & \multicolumn{2}{|c|}{$S_{2222}\left(\mathrm{eV} / \AA^{2}\right)$} & \multicolumn{2}{|c|}{$S_{1122}\left(\mathrm{eV} / \AA^{2}\right)$} & \multicolumn{2}{|c|}{$S_{2211}\left(\mathrm{eV} / \AA^{2}\right)$} & \multicolumn{2}{|c|}{$S_{1212}\left(\mathrm{eV} / \AA^{2}\right)$} \\
\hline & UR & $\mathrm{R}$ & UR & $\mathrm{R}$ & UR & $\mathrm{R}$ & UR & $\mathrm{R}$ & UR & $\mathrm{R}$ \\
\hline $\mathrm{Ag}$ & -0.733 & -0.216 & -0.733 & -0.216 & -0.397 & 0.119 & -0.397 & 0.119 & -0.105 & -0.105 \\
\hline $\mathrm{Al}(\mathrm{EA})$ & -0.089 & -0.565 & -0.089 & -0.565 & 0.695 & 0.218 & 0.695 & 0.218 & 0.214 & 0.214 \\
\hline $\mathrm{Al}(\mathrm{V})$ & 0.138 & -0.193 & 0.138 & -0.193 & 0.396 & 0.066 & 0.396 & 0.066 & -0.072 & -0.072 \\
\hline $\mathrm{Au}$ & -0.901 & -0.329 & -0.901 & -0.329 & -0.730 & -0.158 & -0.730 & -0.158 & -0.247 & -0.247 \\
\hline $\mathrm{Cu}$ & -0.885 & -0.260 & -0.885 & -0.260 & -0.356 & 0.269 & -0.356 & 0.269 & -0.063 & -0.063 \\
\hline $\mathrm{Ni}$ & -1.046 & -0.304 & -1.046 & -0.304 & -0.148 & 0.593 & -0.148 & 0.593 & 0.012 & 0.012 \\
\hline $\mathrm{Pd}$ & -1.185 & -0.402 & -1.185 & -0.402 & -0.778 & 0.006 & -0.778 & 0.006 & -0.231 & -0.231 \\
\hline $\mathrm{Pt}$ & -1.561 & -0.555 & -1.561 & -0.555 & -1.183 & -0.177 & -1.183 & -0.177 & -0.389 & -0.389 \\
\hline
\end{tabular}


TABLE IV. Surface stress tensor of the (111) crystal face. The "1" direction corresponds to [1 10$]$ and "2" corresponds to [11 2 ]. "UR" stands for unrelaxed results and " $\mathrm{R}$ " denotes values for relaxed surfaces. Relaxation here denotes additional relaxation due to strain.

\begin{tabular}{|c|c|c|c|c|c|c|c|}
\hline \multirow[b]{2}{*}{ Element } & \multirow[b]{2}{*}{$\gamma\left(\mathrm{eV} / \AA^{2}\right)$} & \multicolumn{2}{|c|}{$\tau_{11}^{0}\left(\mathrm{eV} / \AA^{2}\right)$} & \multicolumn{2}{|c|}{$\tau_{22}^{0}\left(\mathrm{eV} / \AA^{2}\right)$} & \multicolumn{2}{|c|}{$\tau_{12}^{0}\left(\mathrm{eV} / \AA^{2}\right)$} \\
\hline & & UR & $\mathrm{R}$ & UR & $\mathrm{R}$ & UR & $\mathrm{R}$ \\
\hline $\mathrm{Ag}$ & 0.0499 & 0.0405 & 0.0405 & 0.0405 & 0.0405 & 0.0000 & 0.0000 \\
\hline $\mathrm{Al}(\mathrm{EA})$ & 0.0543 & 0.0568 & 0.0568 & 0.0568 & 0.0568 & 0.0000 & 0.0000 \\
\hline $\mathrm{Al}(\mathrm{V})$ & 0.0515 & 0.0771 & 0.0771 & 0.0771 & 0.0771 & 0.0000 & 0.0000 \\
\hline $\mathrm{Au}$ & 0.0435 & 0.1025 & 0.1025 & 0.1025 & 0.1025 & 0.0000 & 0.0000 \\
\hline $\mathrm{Cu}$ & 0.0775 & 0.0343 & 0.0343 & 0.0343 & 0.0343 & 0.0000 & 0.0000 \\
\hline $\mathrm{Ni}$ & 0.0893 & -0.0072 & -0.0072 & -0.0072 & -0.0072 & 0.0000 & 0.0000 \\
\hline $\mathrm{Pd}$ & 0.0726 & 0.1020 & 0.1020 & 0.1020 & 0.1020 & 0.0000 & 0.0000 \\
\hline $\mathrm{Pt}$ & 0.0773 & 0.1585 & 0.1585 & 0.1585 & 0.1585 & 0.0000 & 0.0000 \\
\hline
\end{tabular}

and the elastic moduli are anisotropic. The results for surface stresses and elastic moduli are given, respectively, in Tables II and III. The surface stresses are all positive and in agreement with previously reported results, ${ }^{18}$ but are somewhat lower in magnitude than those from ab initio calculations as has been seen in previous works. ${ }^{15}$ The surface stresses are also close to the values reported by Streitz et al. ${ }^{21}$ for these materials with similar embedded atom potentials. Also, the surface stresses from unrelaxed calculations and relaxed calculations disagree in only higher decimal places (not shown in the tables). This is not the case with surface elastic constants. In the case of $\mathrm{Al}(\mathrm{V})$, the unrelaxed and relaxed values of $S_{1111}$ disagree in sign. Even in cases where there is agreement in sign, the numerical values disagree by several factors. These results illustrate the profound influence of relaxation on the surface elastic modulus. In all elements, the relaxed values of $S_{1111}\left(=S_{2222}\right)$ are negative for this crystal surface. In the case of $S_{1212}$, the values of $\mathrm{Al}(\mathrm{EA})$ and $\mathrm{Ni}$ are positive while all the remaining elements have negative values. It is also noted that the results for $S_{1212}$ for aluminum differs in sign for the two potentials used. An interesting observation is that surface relaxation has little effect on $S_{1212}$.

\section{B. (111) crystal surface}

The (111) crystal face in fcc crystals has a sixfold symmetry (plane point group $\mathbf{6} \mathbf{m m}$ ) and is therefore isotropic.
The surface stress (Table IV) values are positive for all elements except Ni. The surface elastic constant $S_{1111}$ is positive for $\mathrm{Al}$ (both potentials) and $\mathrm{Ni}$ and negative for the remaining elements (Table V). The values of $S_{1212}$ are positive in the case of $\mathrm{Cu}$ and $\mathrm{Ni}$, and negative in all other cases. As in the case of the (001) surface, the values of surface stress from both unrelaxed and relaxed calculations agree. The elastic constant $S_{1212}$ is not independent; it satisfies the relation $2 S_{1212}=S_{1111}-S_{1122}$ (Table I) which is readily verified from Table V. Due to this reason, the elastic constant $S_{1212}$ is also affected by surface relaxations.

\section{C. (110) crystal surface}

This crystal face, belonging to the plane point group $\mathbf{2 m m}$, is anisotropic and has been studied previously. ${ }^{12}$ The stresses along the [001] direction and the [1 $\overline{10}]$ direction are unequal (Table VI). In the case of Ni the stress in the latter direction is negative. There does not appear to be any trend in magnitudes of the stresses in the two directions. Elastic constants $S_{1111}$ and $S_{2222}$ differ and in the case of $\mathrm{Al}(\mathrm{EA})$ have a different sign (Table VII). Except in case of Pd and $\mathrm{Pt}$, the value of $S_{1111}$ is greater in magnitude that $S_{2222}$. The elastic constant $S_{1122}$ is unequal to $S_{2211}$. Both of these are negative for all elements and agree in sign. The elastic constant representing the shear resistance of this surface is also

TABLE V. Surface elastic modulus tensor of the (111) crystal face. The "1" direction corresponds to [1 $\overline{1} 0]$ and " 2 " corresponds to [112] "UR" stands for unrelaxed results and "R" denotes values for relaxed surfaces. Relaxation here denotes additional relaxation due to strain.

\begin{tabular}{|c|c|c|c|c|c|c|c|c|c|c|}
\hline \multirow[b]{2}{*}{ Element } & \multicolumn{2}{|c|}{$S_{1111}\left(\mathrm{eV} / \AA^{2}\right)$} & \multicolumn{2}{|c|}{$S_{2222}\left(\mathrm{eV} / \AA^{2}\right)$} & \multicolumn{2}{|c|}{$S_{1122}\left(\mathrm{eV} / \AA^{2}\right)$} & \multicolumn{2}{|c|}{$S_{2211}\left(\mathrm{eV} / \AA^{2}\right)$} & \multicolumn{2}{|c|}{$S_{1212}\left(\mathrm{eV} / \AA^{2}\right)$} \\
\hline & UR & $\mathrm{R}$ & UR & $\mathrm{R}$ & UR & $\mathrm{R}$ & UR & $\mathrm{R}$ & UR & $\mathrm{R}$ \\
\hline $\mathrm{Ag}$ & -0.487 & -0.190 & -0.487 & -0.190 & -0.306 & -0.128 & -0.306 & -0.128 & -0.090 & -0.031 \\
\hline $\mathrm{Al}(\mathrm{EA})$ & 0.291 & 0.283 & 0.291 & 0.283 & 0.322 & 0.354 & 0.322 & 0.354 & -0.015 & -0.036 \\
\hline $\mathrm{Al}(\mathrm{V})$ & 0.107 & 0.005 & 0.107 & 0.005 & 0.339 & 0.241 & 0.339 & 0.241 & -0.116 & -0.118 \\
\hline $\mathrm{Au}$ & -0.995 & -0.498 & -0.995 & -0.498 & -0.618 & -0.169 & -0.618 & -0.169 & -0.188 & -0.164 \\
\hline $\mathrm{Cu}$ & -0.448 & -0.123 & -0.448 & -0.123 & -0.290 & -0.140 & -0.290 & -0.140 & -0.079 & 0.009 \\
\hline $\mathrm{Ni}$ & -0.199 & 0.136 & -0.199 & 0.136 & -0.116 & -0.085 & -0.116 & -0.085 & -0.042 & 0.111 \\
\hline $\mathrm{Pd}$ & -1.019 & -0.488 & -1.019 & -0.488 & -0.643 & -0.237 & -0.643 & -0.237 & -0.188 & -0.125 \\
\hline $\mathrm{Pt}$ & -1.577 & -0.791 & -1.577 & -0.791 & -0.980 & -0.306 & -0.980 & -0.306 & -0.299 & -0.243 \\
\hline
\end{tabular}


TABLE VI. Surface stress tensor of the (110) crystal face. The "1" direction corresponds to [001] and "2" corresponds to [1 10$]$ ]. "UR" stands for unrelaxed results and "R" denotes values for relaxed surfaces. Relaxation here denotes additional relaxation due to strain.

\begin{tabular}{|c|c|c|c|c|c|c|c|}
\hline \multirow[b]{2}{*}{ Element } & \multirow[b]{2}{*}{$\gamma\left(\mathrm{eV} / \AA^{2}\right)$} & \multicolumn{2}{|c|}{$\tau_{11}^{0}\left(\mathrm{eV} / \AA^{2}\right)$} & \multicolumn{2}{|c|}{$\tau_{22}^{0}\left(\mathrm{eV} / \AA^{2}\right)$} & \multicolumn{2}{|c|}{$\tau_{12}^{0}\left(\mathrm{eV} / \AA^{2}\right)$} \\
\hline & & UR & $\mathrm{R}$ & UR & $\mathrm{R}$ & UR & $\mathrm{R}$ \\
\hline $\mathrm{Ag}$ & 0.0585 & 0.0436 & 0.0436 & 0.0403 & 0.0403 & 0.0000 & 0.0000 \\
\hline $\mathrm{Al}(\mathrm{EA})$ & 0.0647 & 0.0732 & 0.0732 & 0.1007 & 0.1007 & 0.0000 & 0.0000 \\
\hline $\mathrm{Al}(\mathrm{V})$ & 0.0598 & 0.0811 & 0.0811 & 0.0936 & 0.0936 & 0.0000 & 0.0000 \\
\hline $\mathrm{Au}$ & 0.0529 & 0.0561 & 0.0561 & 0.0918 & 0.0918 & 0.0000 & 0.0000 \\
\hline $\mathrm{Cu}$ & 0.0889 & 0.0621 & 0.0621 & 0.0373 & 0.0373 & 0.0000 & 0.0000 \\
\hline $\mathrm{Ni}$ & 0.1028 & 0.0504 & 0.0504 & -0.0036 & -0.0036 & 0.0000 & 0.0000 \\
\hline $\mathrm{Pd}$ & 0.0869 & 0.0773 & 0.0773 & 0.0977 & 0.0977 & 0.0000 & 0.0000 \\
\hline $\mathrm{Pt}$ & 0.0944 & 0.0937 & 0.0937 & 0.1463 & 0.1463 & 0.0000 & 0.0000 \\
\hline
\end{tabular}

negative for all elements. As in the case of the (001) surface, the surface stresses and the elastic constant $S_{1212}$ are unaffected by relaxation.

\section{D. (112) crystal surface}

As in the case of the (110) crystal face this surface also belongs to the $\mathbf{2} \mathbf{m m}$ point group and hence is anisotropic. The surface stresses are positive in all cases (Table VIII). The elastic constants (Table IX) $S_{1111}$ and $S_{2222}$ are all of negative sign except in the case of Ni. Also, $S_{2222}$ along this surface has a comparatively large magnitude compared to moduli on other surfaces. In most cases $S_{1122}$ is close in value to $S_{2211}$, this surface is elastically less anisotropic than the (110) surface. The elastic constant $S_{1212}$ is negative in all cases. Again, the surface stresses are insensitive to surface relaxations. The elastic modulus $S_{1212}$ is strongly dependent on relaxations.

\section{E. Comparative study and trends}

It is of interest to make a comparative study of the elastic properties with the purpose of uncovering trends in their behavior with respect to surfaces and elements. To achieve this comparison, two quantities pertaining to elastic modulus tensor are considered. First is the "surface bulk modulus" which is equal to $S_{i i j j}$ and gives a measure of the resistance of the surface to homogeneous dilatation, and second is the surface shear elastic constant $S_{1212}$. The comparative information is collected in Tables X and XI. Elements are grouped according to their positions in the periodic table. $\mathrm{Al}(\mathrm{EA})$ and $\mathrm{Al}(\mathrm{V})$ are considered first, followed by the noble metals $\mathrm{Cu}, \mathrm{Ag}$, and $\mathrm{Au}$, and the $\mathrm{Ni}$ group metals $\mathrm{Ni}, \mathrm{Pd}$, and $\mathrm{Pt}$.

From Table $\mathrm{X}$ it is evident that two potentials for $\mathrm{Al}$ roughly produce the same general trends for surface bulk modulus. In the case of $\mathrm{Al}(\mathrm{EA})$, the surface bulk modulus is ordered as $(111)>(001)>(110)>(112)$, while in case of $\mathrm{Al}(\mathrm{V})$, the order is $(111)>(001)>(112)>(110)$. In case of noble metals, $\mathrm{Cu}$ and $\mathrm{Ag}$ have the surface bulk modulus in the order $(001)>(111)>(112)>(110)$. Au, the last element in this group, deviates and has the ordering as (110) $>(001)>(111)>(112)$. Interestingly, the first two elements in the $\mathrm{Ni}$ group, $\mathrm{Ni}$ and $\mathrm{Pd}$, have surface bulk modulus ordered as $(001)>(111)>(112)>(110)$, the same as the first two noble metals. Additionally, $\mathrm{Pt}$, the last element in the $\mathrm{Ni}$ group, deviates to $(001)>(110)>(111)>(112)$. A further interesting point is seen from the results. The surface bulk modulus for the (001) and (111) decreases on going down

TABLE VII. Surface elastic modulus tensor of the (110) crystal face. The "1" direction corresponds to [001] and " 2 " corresponds to [110]. "UR" stands for unrelaxed results and "R" denotes values for relaxed surfaces. Relaxation here denotes additional relaxation due to strain.

\begin{tabular}{|c|c|c|c|c|c|c|c|c|c|c|}
\hline \multirow[b]{2}{*}{ Element } & \multicolumn{2}{|c|}{$S_{1111}\left(\mathrm{eV} / \AA^{2}\right)$} & \multicolumn{2}{|c|}{$S_{2222}\left(\mathrm{eV} / \AA^{2}\right)$} & \multicolumn{2}{|c|}{$S_{1122}\left(\mathrm{eV} / \AA^{2}\right)$} & \multicolumn{2}{|c|}{$S_{2211}\left(\mathrm{eV} / \AA^{2}\right)$} & \multicolumn{2}{|c|}{$S_{1212}\left(\mathrm{eV} / \AA^{2}\right)$} \\
\hline & UR & $\mathrm{R}$ & UR & $\mathrm{R}$ & UR & $R$ & UR & $\mathrm{R}$ & UR & $\mathrm{R}$ \\
\hline $\mathrm{Ag}$ & -0.788 & -0.571 & -0.533 & -0.429 & -0.508 & -0.357 & -0.505 & -0.354 & -0.228 & -0.228 \\
\hline $\mathrm{Al}(\mathrm{EA})$ & -0.932 & -1.254 & -0.115 & 0.087 & -0.103 & -0.067 & -0.130 & -0.094 & -0.063 & -0.063 \\
\hline $\mathrm{Al}(\mathrm{V})$ & 0.066 & -0.507 & 0.190 & -0.182 & 0.376 & -0.081 & 0.364 & -0.094 & -0.098 & -0.098 \\
\hline $\mathrm{Au}$ & -0.790 & -0.185 & -0.972 & -0.543 & -0.565 & -0.055 & -0.601 & -0.090 & -0.118 & -0.118 \\
\hline $\mathrm{Cu}$ & -1.045 & -0.908 & -0.511 & -0.445 & -0.647 & -0.550 & -0.622 & -0.525 & -0.352 & -0.352 \\
\hline $\mathrm{Ni}$ & -1.359 & -1.442 & -0.269 & -0.314 & -0.768 & -0.836 & -0.713 & -0.782 & -0.600 & -0.600 \\
\hline $\mathrm{Pd}$ & -1.204 & -0.680 & -1.069 & -0.748 & -0.803 & -0.392 & -0.824 & -0.412 & -0.275 & -0.275 \\
\hline $\mathrm{Pt}$ & -1.457 & -0.562 & -1.597 & -0.998 & -1.013 & -0.279 & -1.066 & -0.331 & -0.262 & -0.262 \\
\hline
\end{tabular}




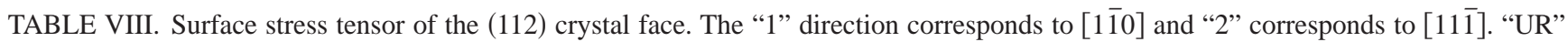
stands for unrelaxed results and " $R$ " denotes values for relaxed surfaces. Relaxation here denotes additional relaxation due to strain.

\begin{tabular}{|c|c|c|c|c|c|c|c|}
\hline \multirow[b]{2}{*}{ Element } & \multirow[b]{2}{*}{$\gamma\left(\mathrm{eV} / \AA^{2}\right)$} & \multicolumn{2}{|c|}{$\tau_{11}^{0}\left(\mathrm{eV} / \AA^{2}\right)$} & \multicolumn{2}{|c|}{$\tau_{22}^{0}\left(\mathrm{eV} / \AA^{2}\right)$} & \multicolumn{2}{|c|}{$\tau_{12}^{0}\left(\mathrm{eV} / \AA^{2}\right)$} \\
\hline & & UR & $\mathrm{R}$ & UR & $\mathrm{R}$ & UR & $\mathrm{R}$ \\
\hline $\mathrm{Ag}$ & 0.0572 & 0.0447 & 0.0447 & 0.0340 & 0.0340 & 0.0000 & 0.0000 \\
\hline $\mathrm{Al}(\mathrm{EA})$ & 0.0633 & 0.0544 & 0.0544 & 0.0681 & 0.0681 & 0.0000 & 0.0000 \\
\hline $\mathrm{Al}(\mathrm{V})$ & 0.0584 & 0.0795 & 0.0795 & 0.0684 & 0.0684 & 0.0000 & 0.0000 \\
\hline $\mathrm{Au}$ & 0.0505 & 0.0835 & 0.0835 & 0.0690 & 0.0690 & 0.0000 & 0.0000 \\
\hline $\mathrm{Cu}$ & 0.0878 & 0.0474 & 0.0474 & 0.0382 & 0.0382 & 0.0000 & 0.0000 \\
\hline $\mathrm{Ni}$ & 0.1013 & 0.0184 & 0.0184 & 0.0129 & 0.0129 & 0.0000 & 0.0000 \\
\hline $\mathrm{Pd}$ & 0.0841 & 0.0947 & 0.0947 & 0.0750 & 0.0750 & 0.0000 & 0.0000 \\
\hline $\mathrm{Pt}$ & 0.0903 & 0.1355 & 0.1355 & 0.1084 & 0.1084 & 0.0000 & 0.0000 \\
\hline
\end{tabular}

the group, both in the Ni group and in noble metals. For the (110) surface the trend is the opposite, the surface bulk modulus increases on going down the group. There is, however, no clear trend in the case of the (112) surface.

A similar study of the surface shear elastic constant can be undertaken from Table XI. Just as in the case of the surface bulk modulus, both the potentials for the case of $\mathrm{Al}$ produce similar trends. The shear modulus is ordered as (001) $>(111)>(110)>(112)$ in the case of $\mathrm{Al}(\mathrm{EA})$ and as $(001)$ $>(110)>(111)>(112)$ in the case of $\mathrm{Al}(\mathrm{V})$. In the case of the noble metals, the shear modulus for the first two, $\mathrm{Cu}$ and $\mathrm{Ag}$, is ordered as $(111)>(001)>(112)>(110)$. Au has a different ordering, $(110)>(112)>(111)>(001)$. This is similar to the case of the surface bulk modulus where the first two elements show the same trend while the last element deviates. In the case of the Ni group elements, each of them show a different trend for the ordering of the shear modulus, although $\mathrm{Ni}$ and $\mathrm{Pd}$ are close in ordering. Finally, the shear modulus of the (001) and (111) decreases on going down the group, in both noble metals and $\mathrm{Ni}$ group. However, the shear modulus increases on going down the group for the case of the (110) surface. In the case of the (112) surface, the shear modulus decreases on going down the group for the noble metals.

\section{F. Conclusions and future directions}

This paper achieves two main objectives. First, a fully nonlinear formulation of surface elasticity is presented. Second, a method for calculation of the surface elastic properties is developed. The most important result uncovered by this work is the importance of accounting for additional surface relaxations engendered by the applied strain. It is found that the relaxation effects do not affect the surface stress tensor and, in the case of some crystal faces, the surface shear elastic constant $S_{1212}$. All other elastic constants are strongly affected by the relaxation, in that relaxed and unrelaxed results can differ even in sign. The limitations of the embedded atom method in describing surfaces and interfaces are well known, and the results in this work are only likely to show trends. In this regard, the study indicates the presence of some general trends as indicated in the previous section. $A b$ initio methods are best suited to determine accurate numerical values of surface elastic constants. The finding in the present study amounts to the conclusion that the relaxations of the atoms (and not just the determination of the ground state wave functions based on the homogeneously strained positions of the atoms) is essential for the accurate determination of surface elastic constants. Also, the accurate determination of the total surface energy as a function of strain

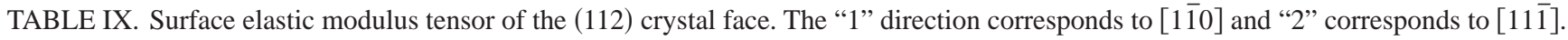
"UR" stands for unrelaxed results and "R" denotes values for relaxed surfaces. Relaxation here denotes additional relaxation due to strain.

\begin{tabular}{|c|c|c|c|c|c|c|c|c|c|c|}
\hline \multirow[b]{2}{*}{ Element } & \multicolumn{2}{|c|}{$S_{1111}\left(\mathrm{eV} / \AA^{2}\right)$} & \multicolumn{2}{|c|}{$S_{2222}\left(\mathrm{eV} / \AA^{2}\right)$} & \multicolumn{2}{|c|}{$S_{1122}\left(\mathrm{eV} / \AA^{2}\right)$} & \multicolumn{2}{|c|}{$S_{2211}\left(\mathrm{eV} / \AA^{2}\right)$} & \multicolumn{2}{|c|}{$S_{1212}\left(\mathrm{eV} / \AA^{2}\right)$} \\
\hline & UR & $\mathrm{R}$ & UR & $\mathrm{R}$ & UR & $\mathrm{R}$ & UR & $\mathrm{R}$ & UR & $\mathrm{R}$ \\
\hline $\mathrm{Ag}$ & -0.570 & -0.160 & -0.766 & -0.759 & -0.375 & -0.169 & -0.364 & -0.159 & -0.098 & -0.164 \\
\hline $\mathrm{Al}(\mathrm{EA})$ & 0.069 & -0.113 & -0.346 & -0.676 & -0.172 & -0.331 & -0.186 & -0.344 & -0.062 & -0.371 \\
\hline $\mathrm{Al}(\mathrm{V})$ & 0.233 & -0.163 & 0.123 & -0.431 & 0.363 & -0.016 & 0.374 & -0.005 & -0.082 & -0.176 \\
\hline $\mathrm{Au}$ & -0.925 & -0.534 & -0.902 & -0.571 & -0.540 & -0.154 & -0.525 & -0.140 & -0.066 & -0.120 \\
\hline $\mathrm{Cu}$ & -0.571 & -0.065 & -0.973 & -1.113 & -0.420 & -0.236 & -0.411 & -0.227 & -0.142 & -0.253 \\
\hline $\mathrm{Ni}$ & -0.406 & 0.205 & -1.263 & -1.693 & -0.357 & -0.288 & -0.351 & -0.283 & -0.223 & -0.391 \\
\hline $\mathrm{Pd}$ & -1.070 & -0.475 & -1.206 & -1.035 & -0.666 & -0.259 & -0.646 & -0.240 & -0.126 & -0.220 \\
\hline $\mathrm{Pt}$ & -1.553 & -0.819 & -1.571 & -1.144 & -0.916 & -0.293 & -0.889 & -0.266 & -0.131 & -0.235 \\
\hline
\end{tabular}


TABLE X. Comparison of "surface bulk modulus" for various surfaces and elements.

\begin{tabular}{ccccc}
\hline \hline & \multicolumn{4}{c}{$S_{i i j i}\left(\mathrm{eV} / \AA^{2}\right)$} \\
\cline { 2 - 5 } Element & $(001)$ & $(111)$ & $(110)$ & $(112)$ \\
\hline \multirow{2}{*}{$\mathrm{Al}(\mathrm{EA})$} & -0.695 & 1.275 & -1.327 & -1.464 \\
$\mathrm{Al}(\mathrm{V})$ & -0.254 & 0.492 & -0.864 & -0.615 \\
$\mathrm{Cu}$ & 0.017 & -0.527 & -2.428 & -1.641 \\
$\mathrm{Ag}$ & -0.193 & -0.636 & -1.711 & -1.247 \\
$\mathrm{Au}$ & -0.975 & -1.335 & -0.872 & -1.398 \\
$\mathrm{Ni}$ & 0.578 & 0.102 & -3.374 & -2.059 \\
$\mathrm{Pd}$ & -0.793 & -1.449 & -2.232 & -2.009 \\
$\mathrm{Pt}$ & -1.465 & -2.194 & -2.170 & -2.521 \\
\hline \hline
\end{tabular}

requires simulations with slabs of various thicknesses, necessarily requiring large computational efforts.

Further efforts are required to bring the subject of elastic properties of crystal surfaces on a firm theoretical footing. Prime among them is the connection to surface phonons. ${ }^{33}$ The bulk elastic constants can be derived from the bulk phonon spectrum using the method of long waves. ${ }^{34}$ An important and interesting task is to derive the surface elastic constants from surface phonons using the method of long waves. Other interesting problems include the determination of temperature dependence and effect of ad-atoms on the surface elastic constants. The determination of temperature dependence of surface elastic constants requires the dynamical treatment of surface phonons and will be taken up in a future publication.

The experimental determination of surface elastic constants is likely to prove to be a challenging task. The determination of the surface stress in itself requires careful experiments. ${ }^{1}$ A suggestion for the possible determination of the surface elastic constant is made here along lines similar to that suggested by Kosevich and Kosevich. ${ }^{35}$ For the sake of simplicity a two-dimensional slab of thickness $h$ (lines that bound the slab are treated as surfaces) is considered, with bulk elastic modulus $C$, surface stress $\tau^{0}$, and surface
TABLE XI. Comparison of surface shear modulus for various surfaces and elements.

\begin{tabular}{ccccc}
\hline \hline & \multicolumn{4}{c}{$S_{1212}\left(\mathrm{eV} / \AA^{2}\right)$} \\
\cline { 2 - 5 } Element & $(001)$ & $(111)$ & $(110)$ & $(112)$ \\
\hline $\mathrm{Al}(\mathrm{EA})$ & 0.214 & -0.036 & -0.063 & -0.371 \\
$\mathrm{Al}(\mathrm{V})$ & -0.072 & -0.118 & -0.098 & -0.176 \\
$\mathrm{Cu}$ & -0.063 & 0.009 & -0.352 & -0.253 \\
$\mathrm{Ag}$ & -0.105 & -0.031 & -0.228 & -0.164 \\
$\mathrm{Au}$ & -0.247 & -0.164 & -0.118 & -0.120 \\
$\mathrm{Ni}$ & 0.012 & 0.111 & -0.600 & -0.391 \\
$\mathrm{Pd}$ & -0.231 & -0.125 & -0.275 & -0.220 \\
$\mathrm{Pt}$ & -0.389 & -0.243 & -0.262 & -0.235 \\
\hline \hline
\end{tabular}

elastic constant $S$. When the slab is a few atomic layers thick, the lattice parameter will be unequal to the bulk lattice parameter, i.e., the lattice will experience a strain. A simple calculation shows that the strain $\epsilon_{r}$ can be obtained as

$$
\frac{1}{\epsilon_{r}}=-\left(\frac{C}{2 \tau_{0}} h+\frac{S}{\tau^{0}}\right) .
$$

The experiment to be performed will include determination of $\epsilon_{r}$ for slabs of various thicknesses, and calculation of $S$ and $\tau^{0}$ from Eq. (24) with the knowledge of the bulk elastic constant $C$. Haiss ${ }^{1}$ has pointed out the difficulties involved in such experiments.

\section{ACKNOWLEDGMENTS}

Support for this work by the Indian National Science Academy under the INSA Young Scientist Programme is gratefully acknowledged. The author thanks Narayanan Ravishankar for many stimulating discussions, valuable suggestions, and particularly for pointing out an inconsistency in the first draft of this paper. Discussions with Shobana Narasimhan and Umesh Waghmare are thankfully acknowledged.
*Email address: shenoy@mrc.iisc.ernet.in

${ }^{1}$ W. Haiss, Rep. Prog. Phys. 64, 591 (2001).

${ }^{2}$ R. C. Cammarata, Prog. Surf. Sci. 46, 1 (1994).

${ }^{3}$ U. Harten, A. M. Lahee, J. P. Toennies, and C. Wöll, Phys. Rev. Lett. 54, 2619 (1985).

${ }^{4}$ K. Takayanagi, Y. Tanishiro, K. Yagi, and K. Kobayashi, Surf. Sci. 205, 637 (1988).

${ }^{5}$ J. V. Barth, H. Brune, R. J. Behm, and G. Ertel, Phys. Rev. B 42, 9307 (1990).

${ }^{6}$ R. Sandy, S. G. Morchie, D. M. Zehner, G. Grübel, K. G. Haung, and D. Gibbs, Phys. Rev. Lett. 68, 2192 (1992).

${ }^{7}$ M. Bott, T. Michely, and G. Comsa, Phys. Rev. Lett. 70, 1489 (1993).

${ }^{8}$ R. J. Needs, M. J. Godfrey, and M. Mansfield, Surf. Sci. 242, 215
(1991)

${ }^{9}$ S. Narasimhan and D. Vanderbilt, Phys. Rev. Lett. 69, 1564 (1992).

${ }^{10}$ R. D. Maede and D. Vanderbilt, Phys. Rev. Lett. 63, 1404 (1989).

${ }^{11}$ R. J. Needs, Phys. Rev. Lett. 58, 53 (1987).

${ }^{12}$ P. J. Feibelman, Phys. Rev. B 51, 17867 (1995).

${ }^{13}$ P. J. Feibelman, Phys. Rev. B 56, 2175 (1997).

${ }^{14}$ G. J. Ackland and M. W. Finnis, Philos. Mag. A 54, 301 (1986).

${ }^{15}$ P. Gumbsch and M. S. Daw, Phys. Rev. B 44, 3934 (1991).

${ }^{16}$ R. Gao, Z. L. Wang, Z. Bai, W. A. de Heer, L. Dai, and M. Gao, Phys. Rev. Lett. 85, 622 (2000).

${ }^{17}$ J. Yang, T. Ono, and M. Esashi, J. Vac. Sci. Technol. B 19, 551 (2001).

${ }^{18}$ R. E. Miller and V. B. Shenoy, Nanotechnology 11, 139 (2000). 
${ }^{19}$ V. B. Shenoy, Int. J. Solids Struct. 39, 4039 (2002).

${ }^{20}$ R. Shuttleworth, Proc. Phys. Soc., London, Sect. A 63, 444 (1950).

${ }^{21}$ F. H. Streitz, R. C. Cammarata, and K. Sieradzki, Phys. Rev. B 49, 10699 (1994).

${ }^{22}$ F. H. Streitz, R. C. Cammarata, and K. Sieradzki, Phys. Rev. B 49, 10707 (1994).

${ }^{23}$ M. S. Daw and M. I. Baskes, Phys. Rev. B 29, 6443 (1984).

${ }^{24}$ M. W. Finnis and J. E. Sinclair, Philos. Mag. A 50, 45 (1984).

${ }^{25}$ P. Chadwick, Continuum Mechanics (Wiley, New York, 1976).

${ }^{26}$ M. C. Payne, M. P. Teter, D. C. Allan, T. A. Arias, and J. D. Joannopoulos, Rev. Mod. Phys. 64, 1045 (1992).

${ }^{27}$ P. Nozieres and D. E. Wolf, Z. Phys. B: Condens. Matter 70, 399
(1987).

${ }^{28}$ P. J. Feibelman, Phys. Rev. B 44, 3916 (1991).

${ }^{29}$ O. H. Nielsen and R. M. Martin, Phys. Rev. Lett. 50, 697 (1983).

${ }^{30}$ F. Ercolessi and J. B. Adams, Europhys. Lett. 26, 583 (1994).

${ }^{31}$ A. F. Voter, Intermetallic Compounds: Vol. 1, Principles (Wiley, New York, 1994), pp. 77-90.

${ }^{32}$ D. J. Oh and R. A. Johnson, J. Mater. Res. 3, 471 (1988).

${ }^{33}$ R. F. Wallis, Surf. Sci. 299/300, 612 (1994).

${ }^{34}$ M. Born and K. Huang, Dynamical Theory of Crystal Lattices (Oxford University Press, Oxford, England, 1954).

${ }^{35}$ Y. A. Kosevich and A. M. Kosevich, Solid State Commun. 70, 541 (1989). 